\title{
Differential expression pattern of the proteome in response to cadmium stress based on proteomics analysis of wheat roots
}

Mingyang Jian ${ }^{1 \dagger}$, Dazhong Zhang ${ }^{1+}$, Xiaoying Wang ${ }^{1}$, Shuwei Wei ${ }^{1}$, Yue Zhao ${ }^{1}$, Qin Ding ${ }^{2^{*}}$, Yucui Han ${ }^{1 *}$ and Lingjian $\mathrm{Ma}^{1 *}$

\begin{abstract}
Background: Heavy metal cadmium (Cd) is a common environmental pollutant in soils, which has an negative impacts on crop growth and development. At present, cadmium has become a major soil and water heavy metal pollutant, which not only causes permanent and irreversible health problems for humans, but also causes a significant reduction in crop yields.

Results: This study examined the chemical forms of Cd in the roots of two wheat varieties (M1019 and Xinong20) by continuous extraction and analyzed differences in distribution characteristics of $\mathrm{Cd}$ in the root cell wall, cytoplasm, and organelles by elemental content determination and subcellular separation. Furthermore, we conducted proteomics analysis of the roots of the two varieties under $\mathrm{Cd}$ pollution using mass spectrometry quantitative proteomics techniques. A total of 11,651 proteins were identified, of which 10,532 proteins contained quantitative information. In addition, the differentially expressed proteins in the two varieties were related to DNA replication and repair, protein metabolism, and the glutathione metabolism pathway.
\end{abstract}

Conclusion: The results of this study improve our understanding of the mechanism of plant responses to Cd stress.

Keywords: Cd stress, Wheat roots, Chemical forms, Proteomics analysis

\section{Background}

The harmful effects of nonliving factors on organisms in a specific environment are called abiotic stress [1]. In the natural environment, abiotic stresses including heat, cold, and heavy metals are not only harmful to the environment, but also detrimental to plants and agriculture [2, 3]. Recent studies have shown that environmental pollution, such as heavy metal pollution, poses a serious threat to living organisms. Plants can cause dysfunction of plant

\footnotetext{
* Correspondence: dingqin@nwsuaf.edu.cn; 455205716@qq.com; malingjian@nwsuaf.edu.cn

${ }^{+}$Mingyang Jian and Dazhong Zhang contributed equally to this work.

${ }^{2}$ College of Horticulture, Northwest A\&F University, Yangling 712100, China

${ }^{1}$ College of Agronomy, Northwest A\&F University, Yangling 712100, China
}

proteins under heavy metal stress [4]. With the intensification of human activities such as mining and industrial activities and the excessive using of phosphate fertilizer, cadmium is released into the natural environment through geological and human activities. Even at low concentrations, cadmium is one of the most toxic elements, which is due to high fluidity and bioavailability. Therefore, cadmium can more easily be absorbed in the underground part of plants and accumulated in the aerial part $[5,6]$.

Wheat is one of the most important and the foremost food crops in China. In 2011-2012, wheat production was significantly higher than corn and rice (USDA 2011). Globally, the main food source for human protein intake is wheat, which 
has higher protein content than that of corn and rice [7, 8]. $\mathrm{Cd}$ is a toxic heavy metal that is absorbed by the roots, subsequently inhibiting plant growth and development. Roots are the first organs that are exposed to $\mathrm{Cd}$ ions. Studies have shown that the first defense strategy is to exclude the metal entering the root tissues. Roots rapidly respond to the presence of $\mathrm{Cd}$ by forming a mechanical barrier $[9,10]$. Cadmium, absorbed by roots and accumulated in plants, may lead to physiological, biochemical and structural changes in plants such as free radicals, which directly cause cell peroxidation. It would influence photosynthetic and stimulates leaf apoptosis and necrosis [11-13]. In order to respond cell peroxidation, cadmium can be detoxified by phytochelatin (a glutathione-derived peptide) or use antioxidant enzymes to degrade excess reactive oxygen species (ROS) $[14,15]$. In conclusion, plants adapt to adverse conditions through a series of physiological, cellular, and molecular processes, culminating in stress tolerance [16]. Extensive studies have been conducted on the physiological aspects of plant stress responses, whereas proteomics investigations, particularly involving wheat roots, are limited.

In order to better explain the resistance mechanism of wheat stress on cadmium, proteomics as a useful analysis tools have been used to analyze protein changes due to changes in environmental factors. Proteins are the end products of genes and play an important role in plant cell metabolism and biological processes. With the deepening of proteomics research, they have made an important supplement to gene expression research.

\section{Results}

\section{Distribution of cadmium and DEPs subcellular structure} localization

The combined form of M1019 and Xinong20 Cd is mainly based on the extracted state of sodium chloride
(Fig. 1). The proportion of $\mathrm{Cd}$ in different chemical forms of M1019 variety was sodium chloride extraction state $>$ acetic acid $>$ extraction state $>$ deionized water extraction state $>$ ethanol extraction state $>$ hydrochloric acid extraction state. The proportion of $\mathrm{Cd}$ in different chemical forms of Xinong20 variety is sodium chloride extraction state > deionized water extraction state > ethanol extraction state $>$ acetic acid extraction state $>$ hydrochloric acid extraction state.

From the content of $\mathrm{Cd}$ of subcellular component (Fig. 2), Cd in root tissues of M1019 and Xinong20 was mainly distributed in the cell wall, however the Cd content of M1019 was the lowest in organelles, and the Cd content of Xinong20 was lowest in cytoplasmic. The original statement has been changed: The content of cadmium in the cell wall of M1019 was higher than that of xinong20, and the content of cadmium in the cell fluid and cytoplasm was lower than that of xinong20. In addition, we used wolfpsort, a subcellular localization prediction software, to predict the subcellular localization. The results showed that the upregulation protein that located in cytoplasm of M1019 is a little more than Xinong20. However, the downregulated M1019 located in cytoplasm was approximately half as much as Xinong20. (Figs. 3 and 4). The cytoplasm is the main site for biochemical reactions; therefore, $\mathrm{Cd}$ stresses may imparted greater effects on Xinong20. To further analyze the differentially expressed proteins, we conducted systematic bioinformatics analysis.

\section{Analysis of mass spectrometry data}

We conducted quality control testing of the obtained mass spectrometry data. First, mass errors in all identified peptides were assessed. The results showed that the quality error was centered at 0 and concentrated to a range below $10 \mathrm{PPM}$, indicating that the quality error

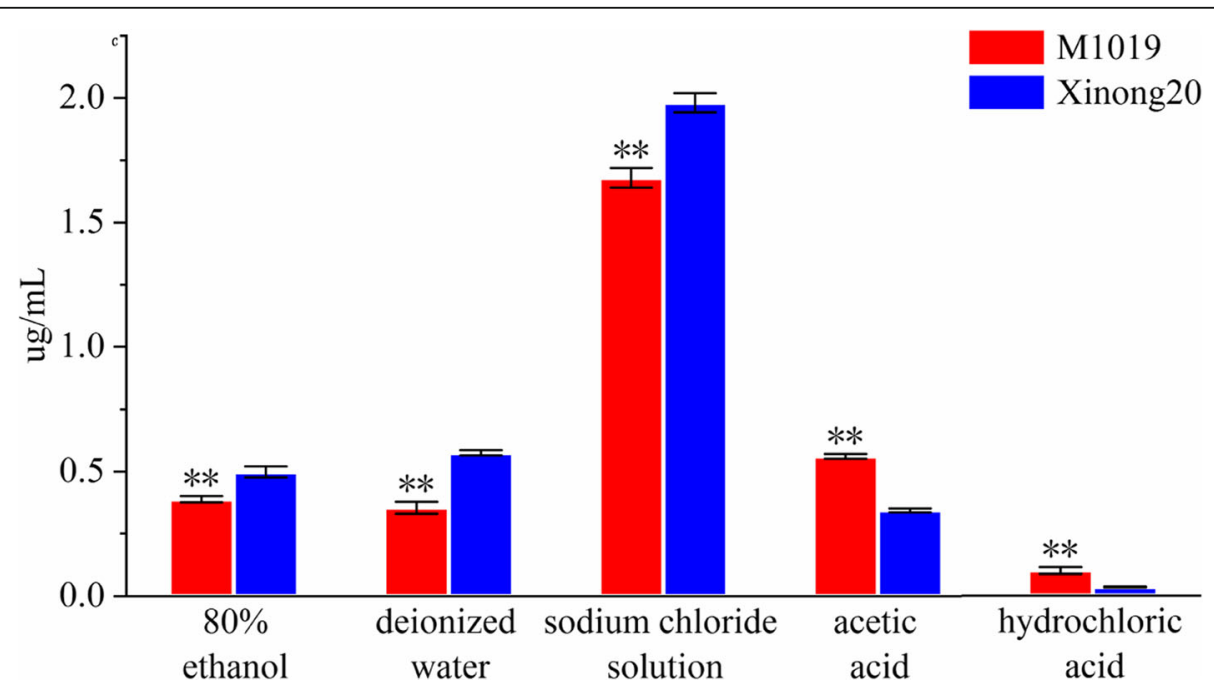

Fig. 1 Cadmium chemical analysis After t-test, the significant differences at the level of 0.01 


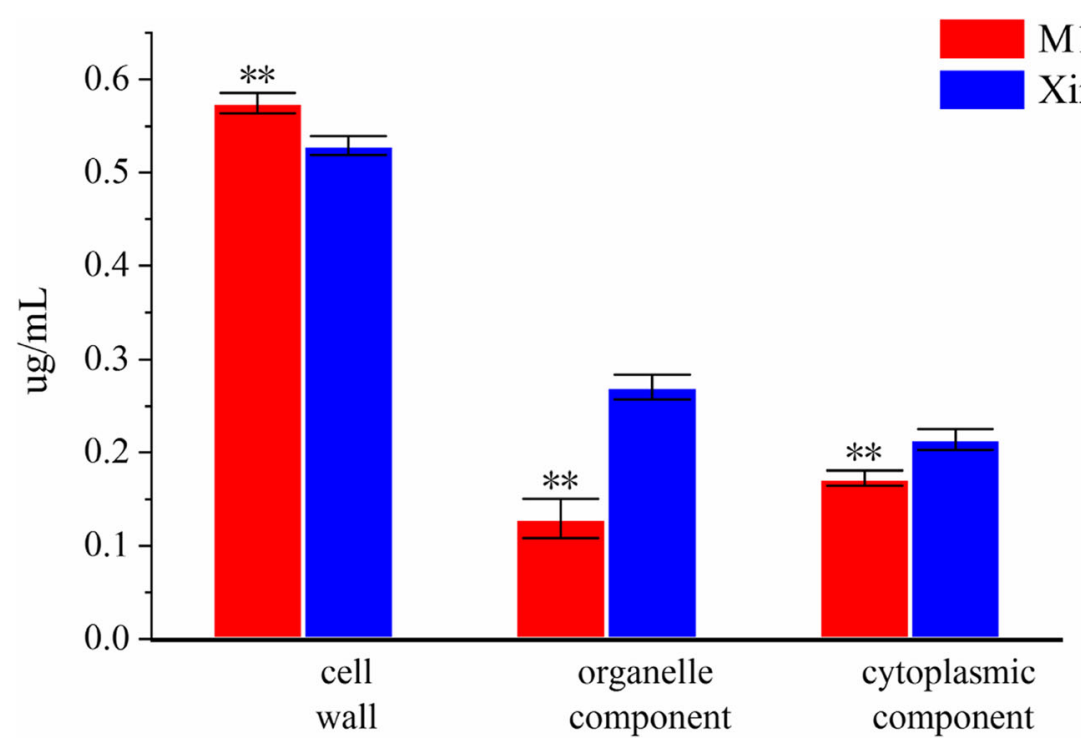

Fig. 2 Distribution of cadmium in the cell wall, organelle components, and cytoplasmic After t-test, the significant difference at the level of 0.01

met the requirements. Second, most of the peptide lengths were distributed between 7 and 16 amino acid residues (Fig. 5). The results showed that the sample conformed to the rule of and the sample preparation reached criterion, so the sample can be used for next step of biological information analysis. Pearson's correlation coefficient was used to determine whether the biological repetition rate was statistically consistent. In this experiment, all sample pairs were used to calculate our Pearson's correlation coefficient. The closer Pearson's coefficient is a measure of the linear correlation between sets of data. When the coefficient closer to -1 is a negative correlation, the closer to 1 is a positive correlation, the closer to 0 is relevant. The results (Fig. 6) showed that the three biological repeats were statistically consistent, indicating that the results were reliable.

A total of 11,651 proteins were identified, of which 10, 532 contained quantitative information. Taking 1.5 times as the change threshold and t-test $p$-value $<0.05$ as the standard, we determined that the expression of 268 proteins in M1019/M1019ck was upregulated and 217 proteins were downregulated. The expression of 684 proteins in Xinong20/Xinong $20_{\text {ck }}$ was upregulated, and that of 680 proteins was downregulated.

\section{Gene ontology enrichment analyses of DEPs}

We conducted GO annotation analysis on the identified differential proteins and explained the biological effects

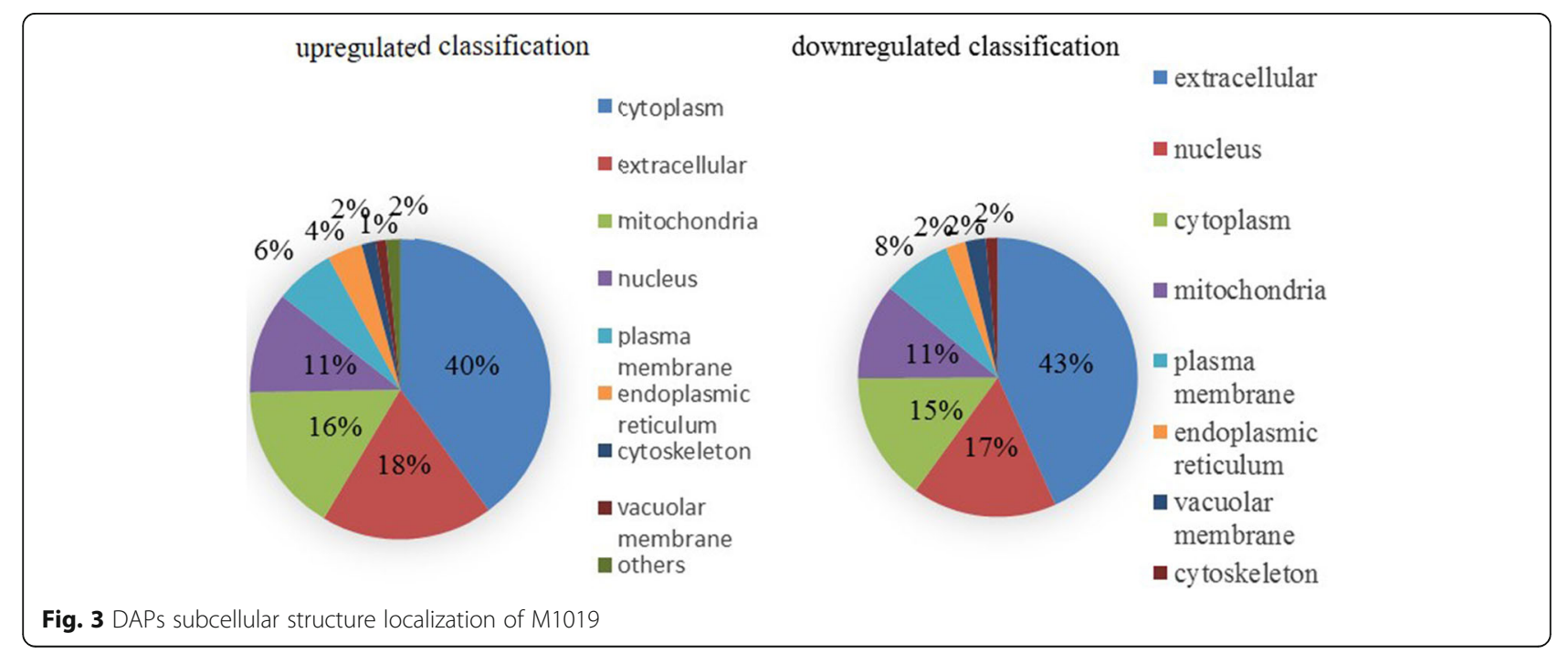




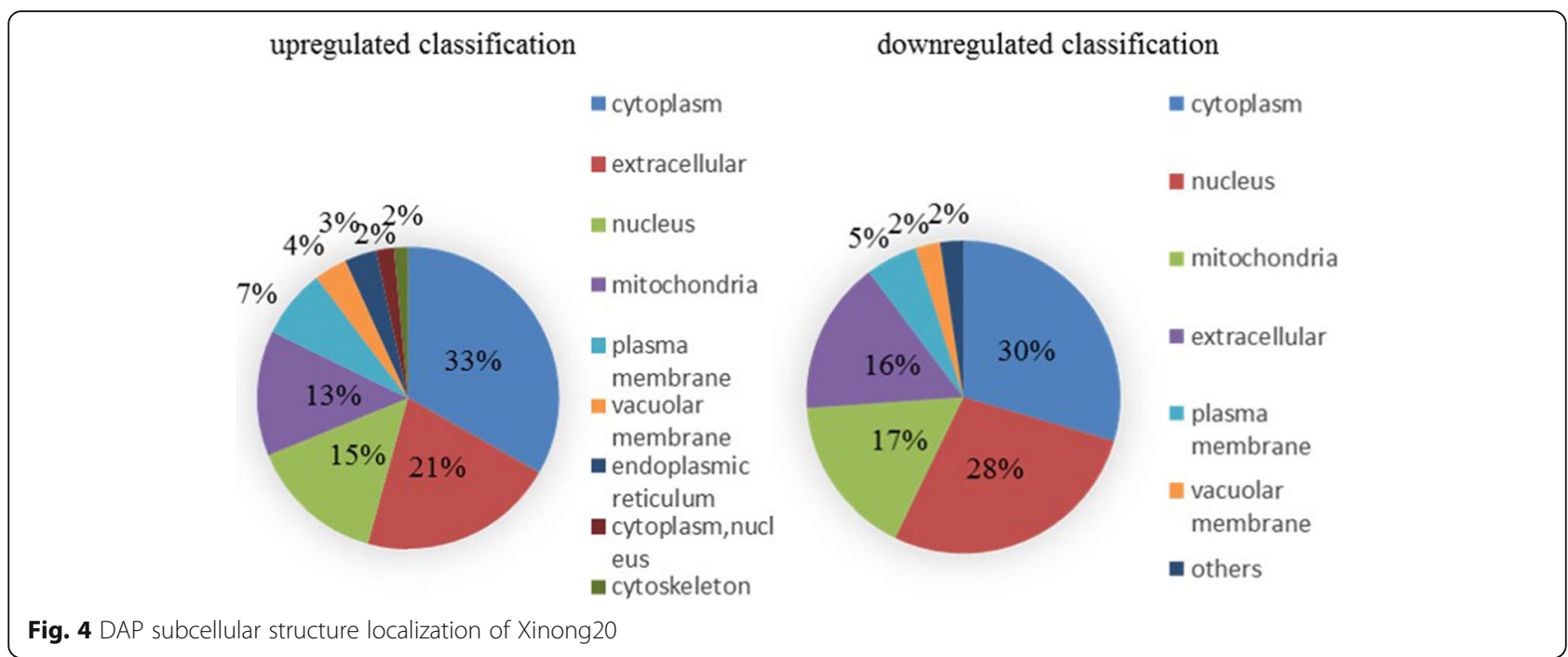

of proteins from different angles. Therefore, we conducted statistical analysis of the distribution of proteins quantified by M1019 and Xinong20 in the GO secondary annotations. The GO annotation is divided into three primary categories: the biological process, the cellular component, and the molecular function (Figs. 7 and 8). Among the biological process functions, the upregulated expression, the DEPs of M1019 were related to responses to stress and carbon fixation. The DEPs of Xinong20 were related to decomposition and amino sugar catabolic process, the cell wall macromolecule catabolic process, polysaccharide catabolic process, regulation of protein catabolic process, and protein ubiquitination. For downregulated expression, the DEPs of

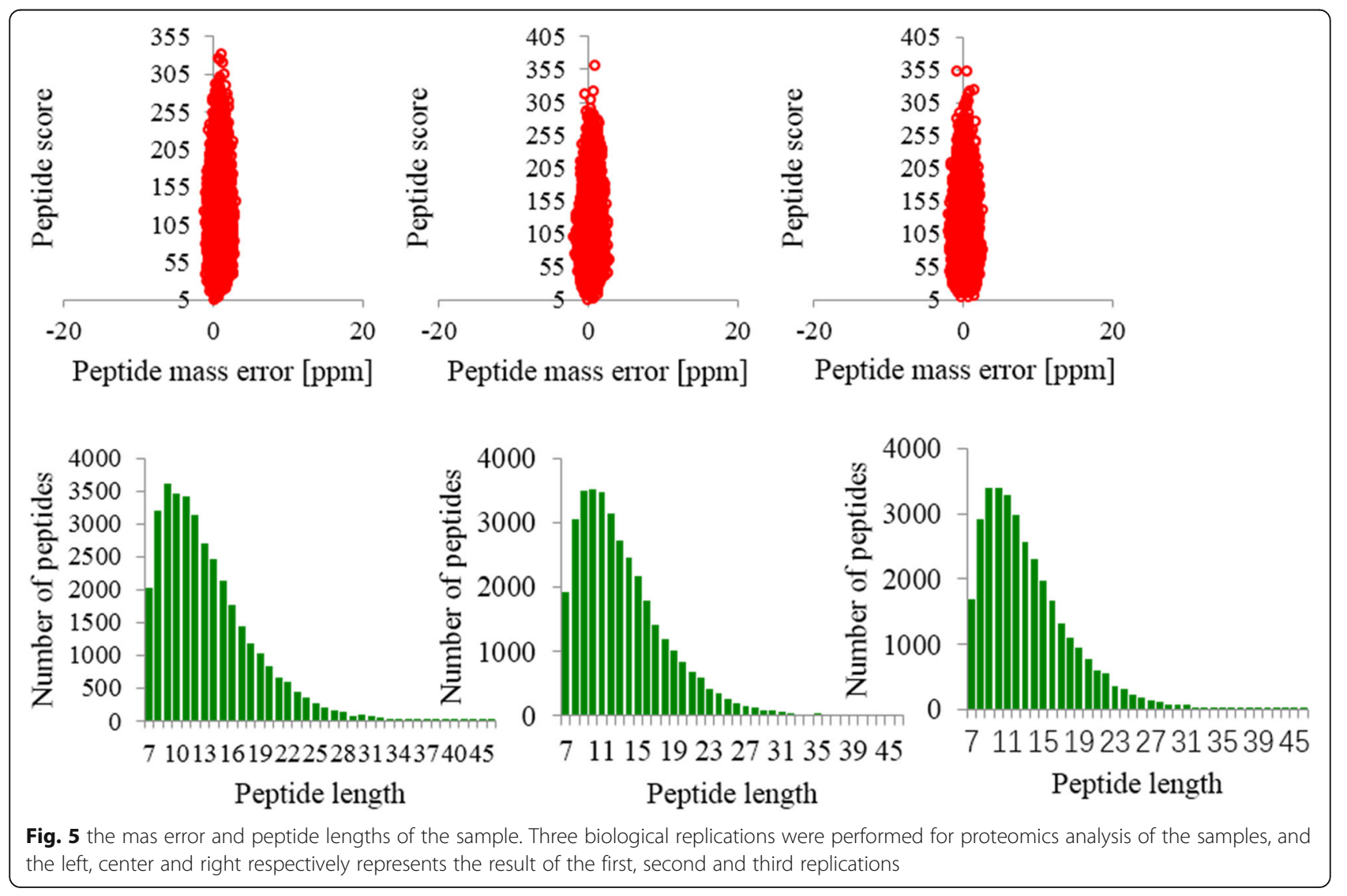




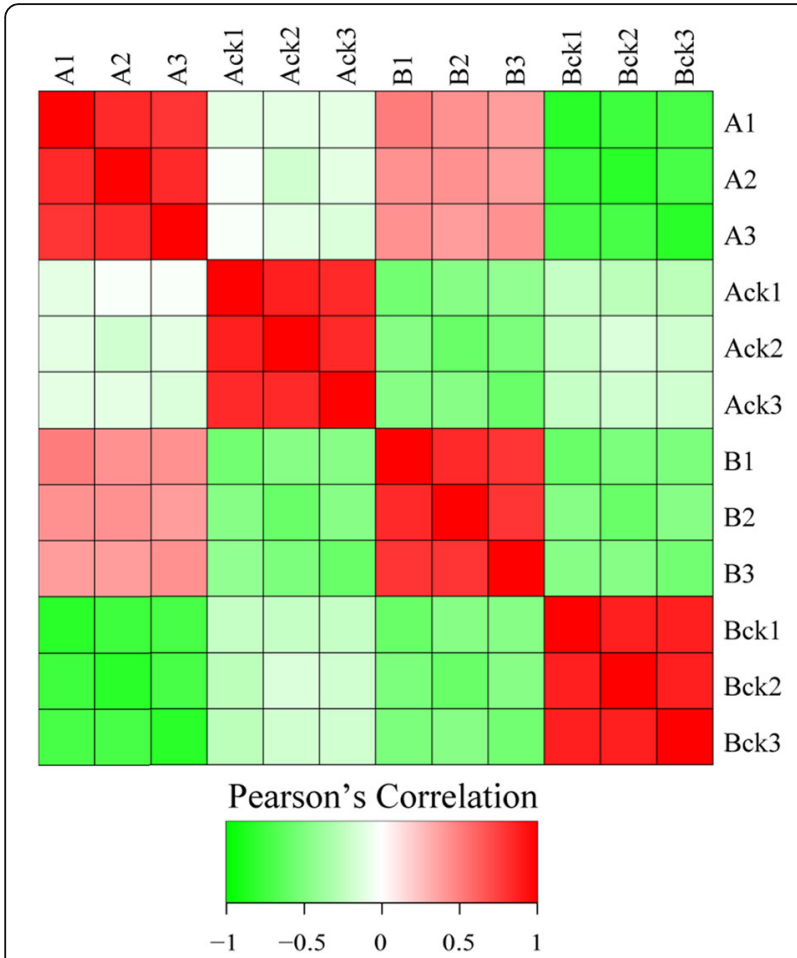

Fig. 6 Heat map draw for calculating our Pearson's Correlation Coefficient using all sample pairs. The A represents wheat M1019, and $A 1, A 2$, and $A 3$ represent the three biological replications of cadmium treatment. Ack,$A_{1} \mathrm{ck}_{2}$ and $\mathrm{Ack}_{3}$ represent three biological replications of the control (nutrient solution only without cadmium). The $B$ represents wheat xinong 20 and B1, B2 and B3 represent three biological replications of cadmium treatment. $B C k_{1}, B C k_{2}$ and $B c k_{3}$ represent three biological replications of the control group (nutrient solution only without cadmium)

M1019 were related to cellular catabolic process. The differentially expressed proteins of Xinong20 were mainly related to the balance of the ion, cell metabolism, and macromolecular biosynthesis. Destruction of ion balance may be a factor that Xinong20 is sensitive to Cd stress.

Among the molecular process functions, the upregulated expression, the DEPs of M1019 were related to transferase activity, transferring glycosyl groups, and metal ion binding. The differentially expressed proteins of Xinong20 were mainly related to peptidase inhibitor activity, including those of endopeptidases. Inversely, the helicase activity of DNA and hydrolase activity, which act on ester bonds, enzyme inhibitor activity, and peptidase inhibitor activity were downregulated. Therefore, the difference in inhibiting enzyme activity will affect peptide synthesis in Xinong20 to some extent, which may indirectly affect protein metabolism process. Among the cellular component process functions, the upregulated expression, the DEPs of M1019 were related to intrinsic and integral components of the membrane. The DEPs of Xinong20 were related to the extracellular region. For the downregulated expression, the DEPs of M1019 were related to external encapsulating structure. The DEPs of Xinong20 were related to ribosomes and ribonucleoprotein complexes (Figs. 7 and 8).

\section{Genomes (KEGG) enrichment analysis of DEPs}

We also made comparisons with the KEGG database to predict possibly important pathways. For upregulated expression, both M1019 and Xinong20 enriched the MAPK signaling pathway-plant and glutathione metabolism. However, the results showed that most of the DEPs in the MAPK signaling pathway of Xinong20 were related to pathogenic proteins. A significantly greater enrichment of the glutathione metabolism pathway of M1019 than that in Xinong20 was observed (Fig. 9). For the upregulated expression, the results indicated that the DEPs of M1019 also enriched in protein processing in endoplasmic reticulum, alpha-linolenic acid metabolism, phenylalanine metabolism, galactose metabolism, and cysteine and methionine metabolism (Table 1).

In terms of downregulated expression, more pathways in Xinong20 were enriched than that observed in M1019. The DEPs related to glycosphingolipid biosynthesis-globo and isoglobo series were highly enriched in M1019 and Xinong20. Moreover, the DEPs related to nitrogen metabolism were enriched in M1019 such as the glutamine synthetase cytosolic isozyme. The DEPs were related to DNA replication, homologous recombination, mismatch repair, and ribosomes. Lysine biosynthesis, nitrogen metabolism, and protein export were also enriched in Xinong20. In terms of protein export pathways, the DEPs in Xinong20 were associated with transport proteins such as the mitochondrial inner membrane protein and signal recognition particle 54 $\mathrm{kDa}$. For the ribosome pathway, numerous DEPs were enriched in Xinong20. In addition, the DNA polymerase delta small subunit and replication protein $32 \mathrm{kDa}$ subunit showed downregulated in the DNA replication, mismatch repair, and homologous recombination pathway (Table 2).

\section{Discussion}

\section{$\mathrm{Cd}$ content distribution and chemical form distribution}

Most of the heavy metal absorption takes place through root cells, which distribute toxic ions to specific organs, tissues, or organelles. Studies have shown that metal ions in Ni-rich plant cells, Zn hyperaccumulators and copper hyperaccumulators are mainly distributed on the cell wall, and the content in organelles is relatively small [17, 18]. In this study, the cadmium distribution of M1019 in root cells was the same as that in Ni-rich plant cells, $\mathrm{Zn}$ hyperaccumulators and copper hyperaccumulators, while the content of cadmium in cytoplasmic component of Xinong20 was higher than that in organelles. Therefore, M1019 can better accumulate cadmium in cell wall, 

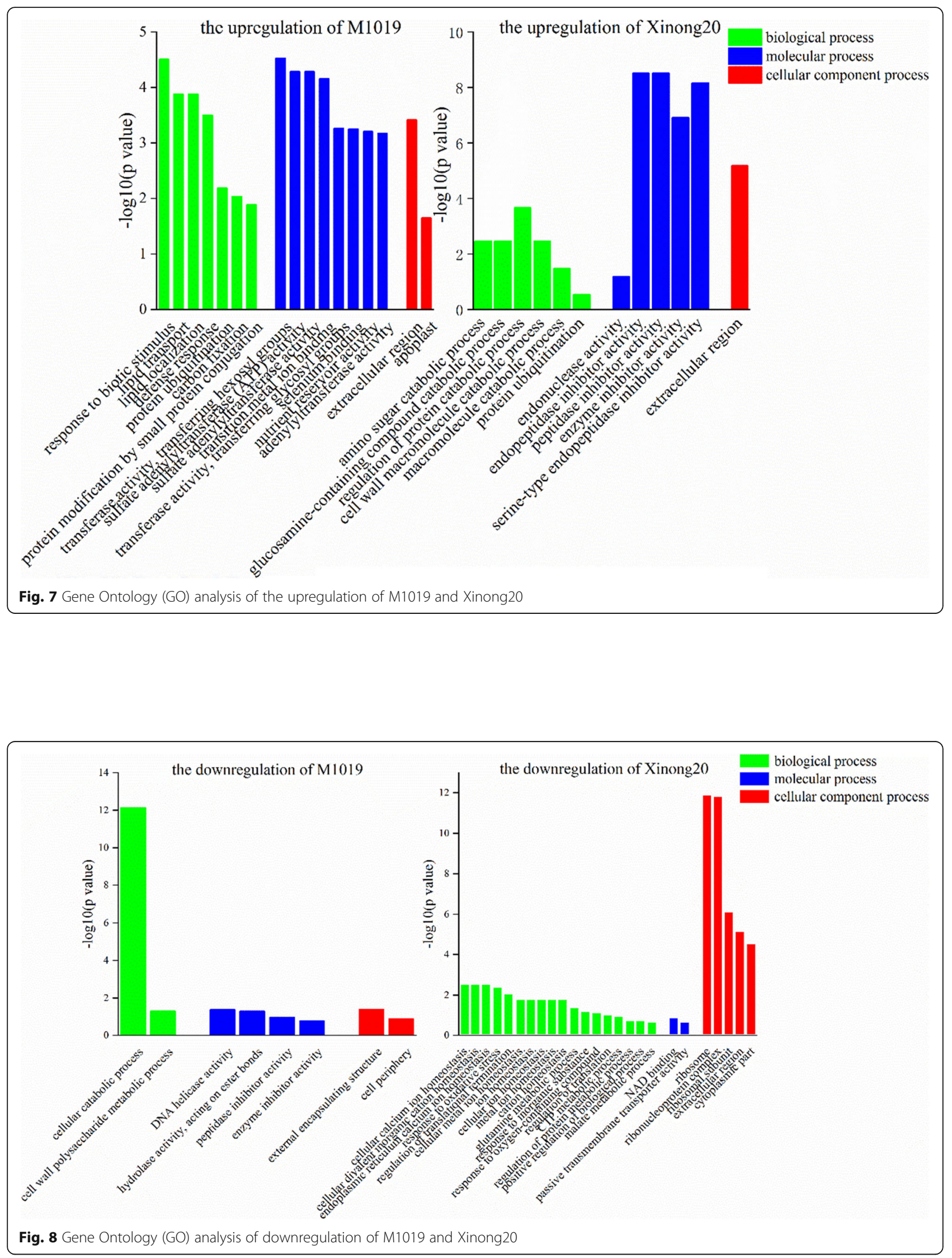


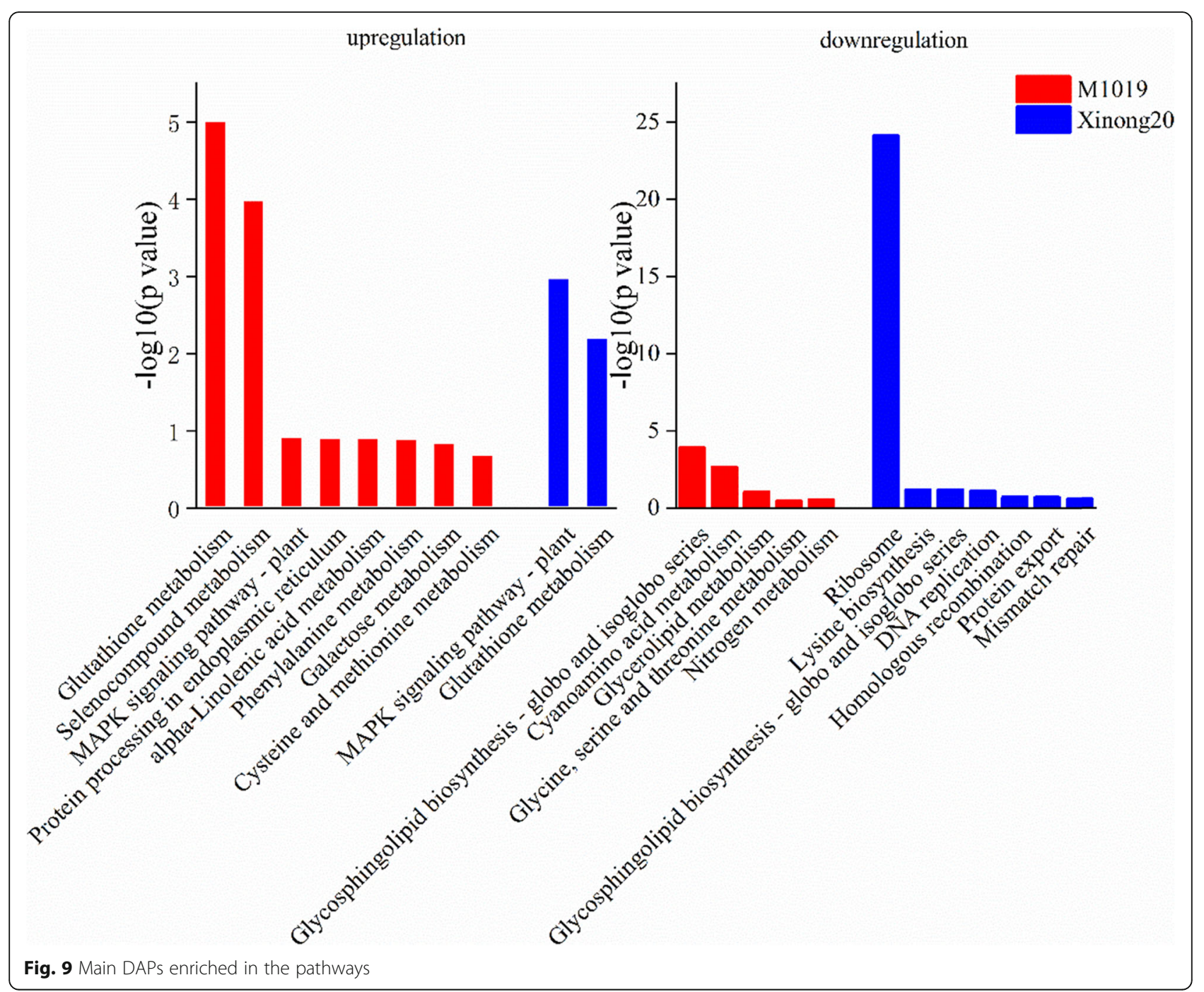

which may be one of the factors of resistance to cadmium in M1019. In addition, the Cd content and percentage of cell organelles of M1019 were lower than Xinong20, and the $\mathrm{Cd}$ content and percentage of cell wall were higher than Xinong20. Therefore, M1019 can more effectively accumulate $\mathrm{Cd}$ in the cell wall and prevent excess $\mathrm{Cd}$ from entering cellular organelles. According to the GO analysis, among the cellular component process, for the downregulated expression, the DEPs were detected in cytoplasmic part. So Xinong20 may be more effected than M1019.

Studies have shown that absorption operations play an important role in maintaining trace amounts of cellular metal ions. Heavy metals can combine with polar compounds to form water-soluble, alcohol-soluble, and acid-soluble low-molecular weight metal chelates to affect heavy metals in plants [19]. Some studies indicate that the ethanol extraction state and water-soluble $\mathrm{Cd}$ have the strongest migration activity, and the acid- soluble (acetic acid and hydrochloric acid extraction) $\mathrm{Cd}$ had the weakest migration activity, so the Cd toxicity effect in the alcohol-soluble and water-soluble form is more significant than other chemical forms on Cd migration activity [20]. In this study, the content of $\mathrm{Cd}$ in the ethanol-extracted and water-extracted state of M1019 variety was lower, and the content of acidsoluble $\mathrm{Cd}$ with weaker migration activity was higher than Xinong20. Acidic extraction with weaker migration activity may also be more conducive to cadmium deposition on the cell wall, thereby slowing down $\mathrm{Cd}$ stress. However, the $\mathrm{Cd}$ with stronger migration activity is more likely to move upwards in Xinong20, thus causing toxicity to the plants. On the other hand, this may contribute to the accumulation of $\mathrm{Cd}$ in Xinong20 plants. We preliminarily concluded that the migration of Cd in M1019 is weaker than that in Xinong20, which may be a key factor for more cell wall deposition in M1019. 
Table 1 The upregulation proteins in M1019 and Xinong20

\begin{tabular}{|c|c|c|}
\hline Wheat cultivars & KEGG pathway & Protein description \\
\hline \multirow[t]{19}{*}{ M1019 } & Cysteine and methionine metabolism & Homocysteine S-methyltransferase 3 \\
\hline & Cysteine and methionine metabolism & 1-aminocyclopropane-1-carboxylate oxidase \\
\hline & Glutathione metabolism & Glutathione S-transferase 4 \\
\hline & Glutathione metabolism & Probable glutathione S-transferase GSTU6 \\
\hline & Glutathione metabolism & Protein IN2-1 homolog B \\
\hline & Glutathione metabolism & Probable glutathione S-transferase GSTU6 \\
\hline & Glutathione metabolism & Probable glutathione S-transferase GSTU6 \\
\hline & Glutathione metabolism & Glutathione S-transferase U18 \\
\hline & Glutathione metabolism & Protein IN2-1 homolog B \\
\hline & Glutathione metabolism & Protein IN2-1 homolog B \\
\hline & Glutathione metabolism & Probable glutathione S-transferase GSTU6 \\
\hline & Glutathione metabolism & Probable glutathione S-transferase GSTU6 \\
\hline & Glutathione metabolism & Probable glutathione S-transferase GSTU6 \\
\hline & Glutathione metabolism & Galactinol--sucrose galactosyltransferase \\
\hline & Glutathione metabolism & Hexokinase-7 \\
\hline & Protein processing in endoplasmic reticulum & Heat shock cognate $70 \mathrm{kDa}$ protein \\
\hline & Protein processing in endoplasmic reticulum & UDP-glucose:glycoprotein glucosyltransferase \\
\hline & Protein processing in endoplasmic reticulum & $17.8 \mathrm{kDa}$ class II heat shock protein \\
\hline & Protein processing in endoplasmic reticulum & Cell division cycle protein 48 homolog \\
\hline \multirow[t]{10}{*}{ Xinong20 } & Glutathione metabolism & Ornithine decarboxylase \\
\hline & Glutathione metabolism & Glutamate--cysteine ligase A, chloroplastic \\
\hline & Glutathione metabolism & Uncharacterized protein \\
\hline & Glutathione metabolism & Probable glutathione S-transferase GSTU6 \\
\hline & Glutathione metabolism & Probable L-ascorbate peroxidase 3 \\
\hline & Glutathione metabolism & 5-oxoprolinase \\
\hline & MAPK signaling pathway - plant & Nucleoside diphosphate kinase 1 \\
\hline & MAPK signaling pathway - plant & Pathogenesis-related protein PRB1-3 \\
\hline & MAPK signaling pathway - plant & Pathogenesis-related protein PRMS \\
\hline & MAPK signaling pathway - plant & Pathogenesis-related protein PRB1-2 \\
\hline
\end{tabular}

\section{Effect of cd stress on protein synthesis}

Protein synthesis is substantially important for normal wheat development, new cell protein formation, protein degradation, and the output balance process. Proteins are synthesized via transcription, translation, posttranslational processing, and modification, which finally lead to the synthesis of the mature protein, which is strictly regulated in multiple steps [21]. Nowadays, classical two-dimensional (2D) gel-based proteomics has been difficult to meet the analysis of large dynamic range due to several technical limitations including gel separation and identification capabilities [22], so we choose proteomics techniques to analysis.

Studies have found that $\mathrm{Cd}$ contamination can cause DNA damage to organisms such as DNA fragmentation and DNA cross-linking, which interferes with the normal expression of genes. In addition, $\mathrm{Cd}^{2+}$ can randomly bind to DNA, resulting in single-strand DNA breaks [23]. Most DNA damage due to metal toxicity involves DNA fragmentation. The MMR system is a major DNA repair pathway in all eukaryotes. Most errors that arise during DNA replication can be corrected by DNA polymerase proofreading or by postreplication MMR [24-26]. DNA recombinant repair protein can repair the damage caused by heavy metal pollution to the DNA molecules in the cells and restores its DNA structure and function. We found that DNA replication, homologous recombination, and mismatch repair pathway in Xinong20 were downregulated. DNA polymerase delta small subunit and replication protein A 32-kDa subunit showed downregulated in the three pathways. DNA polymerase and replication protein are essential to DNA replication. However, no decrease in these proteins was detected in 
Table 2 The downregulation proteins in M1019 and Xinong20

\begin{tabular}{|c|c|c|}
\hline Wheat cultivars & KEGG pathway & Protein description \\
\hline \multirow[t]{18}{*}{ M1019 } & Glycerolipid metabolism & Alpha-galactosidase \\
\hline & Glycosphingolipid biosynthesis - globo and isoglobo series & Alpha-galactosidase \\
\hline & Glycosphingolipid biosynthesis - globo and isoglobo series & Beta-hexosaminidase 3 \\
\hline & Glycosphingolipid biosynthesis - globo and isoglobo series & Alpha-galactosidase \\
\hline & Starch and sucrose metabolism & Beta-xylosidase/alpha-L-arabinofuranosidase 2 \\
\hline & Starch and sucrose metabolism & Beta-xylosidase/alpha-L-arabinofuranosidase 2 \\
\hline & Starch and sucrose metabolism & Beta-xylosidase/alpha-L-arabinofuranosidase 2 \\
\hline & Starch and sucrose metabolism & Beta-xylosidase/alpha-L-arabinofuranosidase 2 \\
\hline & Starch and sucrose metabolism & Endoglucanase 10 \\
\hline & Starch and sucrose metabolism & Beta-fructofuranosidase, insoluble isoenzyme 7 \\
\hline & Cyanoamino acid metabolism & Beta-xylosidase/alpha-L-arabinofuranosidase 2 \\
\hline & Cyanoamino acid metabolism & Beta-xylosidase/alpha-L-arabinofuranosidase 2 \\
\hline & Cyanoamino acid metabolism & Beta-xylosidase/alpha-L-arabinofuranosidase 2 \\
\hline & Cyanoamino acid metabolism & Beta-xylosidase/alpha-L-arabinofuranosidase 2 \\
\hline & Glycine, serine and threonine metabolism & Threonine dehydratase biosynthetic, chloroplastic \\
\hline & Glycine, serine and threonine metabolism & Threonine dehydratase biosynthetic, chloroplastic \\
\hline & Nitrogen metabolism & Glutamine synthetase cytosolic isozyme 1-3 \\
\hline & Nitrogen metabolism & Glutamine synthetase cytosolic isozyme 1-3 \\
\hline \multirow[t]{21}{*}{ Xinong20 } & Protein export & Mitochondrial inner membrane protein OXA1 \\
\hline & Protein export & Signal recognition particle 54 kDa protein 1 \\
\hline & Protein export & Protein transport protein Sec61 subunit beta \\
\hline & Protein export & Signal recognition particle 54 kDa protein 3 \\
\hline & DNA replication & DNA polymerase delta small subunit \\
\hline & DNA replication & DNA replication licensing factor MCM4 \\
\hline & DNA replication & DNA replication licensing factor MCM5 \\
\hline & DNA replication & DNA polymerase delta small subunit \\
\hline & Mismatch repair & Replication protein A $32 \mathrm{kDa}$ subunit A \\
\hline & Homologous recombination & DNA polymerase delta small subunit \\
\hline & Homologous recombination & Replication protein A $32 \mathrm{kDa}$ subunit A \\
\hline & Homologous recombination & $60 S$ ribosomal protein $L 27 a-3$ \\
\hline & Ribosome & $40 \mathrm{~S}$ ribosomal protein S11 \\
\hline & Glycosphingolipid biosynthesis - globo and isoglobo series & Alpha-galactosidase \\
\hline & Glycosphingolipid biosynthesis - globo and isoglobo series & Beta-hexosaminidase 3 \\
\hline & Lysine biosynthesis & Diaminopimelate epimerase, chloroplastic \\
\hline & Spliceosome & Glycine-rich RNA-binding protein RZ1C \\
\hline & Spliceosome & DEAD-box ATP-dependent RNA helicase 14 \\
\hline & Spliceosome & mediator of RNA polymerase II transcription subunit 37e \\
\hline & Spliceosome & Heat shock cognate $70 \mathrm{kDa}$ protein \\
\hline & Spliceosome & Splicing factor U2af small subunit B \\
\hline
\end{tabular}

M1019. Therefore, Cd stress will affect the normal replication of DNA and mismatch repair of Xinong20. DNA is a template for transcription. Thus, DNA replication and repair are disrupted, which is highly likely to cause errors in transcription.
After gene transcription, mRNA precursors contain protein-coding exons and noncoding introns and require splicing by spliceosomes before a mature mRNA is formed. We found that components of the spliceosome pathway were downregulated in Xinong20 such as 
splicing factor U2af small subunit B, serine /argininerich splicing factor RS31, and serine/arginine-rich SC35like splicing factor SCL33. However, no downregulation was detected in M1019. The hn-RNA is the initial product of DNA transcription, which requires further processing and modification of the spliceosome to form mRNAs with coding function. Experimental results indicate that protein expression is further affected by processing of transcriptional primary products that affect mRNA of Xinong20.

Mitochondrial heat shock proteins play a role in the degradation of unstable and misfolded proteins and in the folding and refolding of proteins [27]. When cells are stressed by metal ions, heat shock proteins can treat harmful metal ions by helping other proteins fold. HSP70 on tomato cell membranes act as a molecularchaperones that protect cells from damage [28]. We found that protein processing in the endoplasmic reticulum pathway was upregulated in M1019 such as heat shock cognate $70 \mathrm{kDa}$ protein, $17.8 \mathrm{kDa}$ class II heat shock protein, and cell division cycle protein 48 homolog. In contrast, the heat shock cognate $70 \mathrm{kDa}$ protein and $17.8 \mathrm{kDa}$ class II heat shock protein was downregulated in Xinong20. Heat shock proteins, which are involved in the translation process and in the postprocessing process, were also downregulated in Xinong20. However, this was upregulated in M1019. By affecting the processing of Xinong20 mRNA posttranscriptional proteins, the synthesis of proteins is affected, ultimately affecting the expression of protein function. M1019 can effectively fold proteins to cope with Cd stress.

Approximately $30 \%$ of the eukaryotic proteome is transmitted to the downstream compartments by endoplasmic reticulum (ER) folding [29]. Signal recognition particles (SRPs) are important for targeting secreted proteins in a GTP dependent process, which transmit secreted proteins to the plasma membrane or endoplasmic reticulum [30]. This is critical to the vitality of all organisms [31, 32]. Studies have characterized canine SRP Srp72, Srp68, Srp54, Srpl9, Srpl4, and Srp9 based on their apparent molecular weights. In addition, the Sec61 complex is also the central component of the protein translocation apparatus of the ER membrane [33, 34].

In summary, proteins are products of gene expression. The rate of protein synthesis and transport was lower or abolished in Xinong20. We speculate that this may also be a key factor that indirectly affects the synthesis of macromolecular substances. This ultimately leads to the sensitivity of Xinong20 to Cd toxicity.

\section{Effect of cd stress on glutathione metabolism}

GST plays an important role in plant stress tolerance, which can be induced under a variety of biotic or abiotic stress conditions, catalyzes the binding of glutathione to harmful substances, and transports the polymer to the vacuole through the action of ATP-binding transporter for detoxification [35]. Therefore, the binding and transport of glutathione to toxic substances usually require the participation of glutathione S-transferase (GST). On the other hand, glutamine synthetase (GS) is a key enzyme for plant-catalyzed conversion of glutamate (Glu) to glutamine (Gln). Decreased GS activity can promote the accumulation of Glu and contribute to the biosynthesis of the Cd-detoxifying substance, glutathione. In addition, $A B C$ transporters in plants play a role in detoxification defense by transporting secondary metabolites. Numerous studies have revealed that $A B C$ transporters are also required for normal plant development [36]. In this study, in the upregulation expression, the GST of M1019 and Xinong20 both showed an increase, but the M1019 showed a more increase. In the downregulation expression, the GS of M1019 showed a downregulation expression, but GS of Xinong20 did not show the downregulation expression. So, this will be beneficial for accumulating of glutathione. In addition, $A B C$ was upregulated in M1019. Therefore, glutathione and $A B C$ may be one factor of the insensitivity of M1019 to Cd.

\section{Conclusions}

By comparing the differences in $\mathrm{Cd}$ enrichment between two varieties, we show that the insensitive varieties M1019 can concentrate more Cd in the cell, and the migration activity of $\mathrm{Cd}$ in the roots is relatively weak. In addition, we used proteomics analysis to study wheatrelated proteins under $\mathrm{Cd}$ stress. Our results indicate that insensitive wheat Xinong20 can more fully utilize glutathione for detoxification, and the sensitive variety exhibits weaker DNA replication, protein synthesis and degradation. The in-depth study on the mechanism of $\mathrm{Cd}$ tolerance in wheat is of great significance in breeding.

\section{Methods}

Plant materials, growth conditions, and stress treatments

The seeds of wheat M1019 and Xi'nong20 were surfacesterilized with $1.5 \%(\mathrm{v} / \mathrm{v})$ hydrogen peroxide solution for $20 \mathrm{~min}$, washed with distilled water, and imbibed for 24 h. The M1019 is insensitive to Cd stress and is inbred lines crossed by Xi'nong233 and Luoxin988. The Xi'nong 20 is sensitive to Cd stress. The seeds were obtained from own laboratory. Seeds were then grown in Hoagland's nutrient solution $(\mathrm{pH} 7)$ at $23^{\circ} \mathrm{C}$ under 16 -h light/8-h dark conditions. The culture solution was replaced every 2 days. When the second leaves of the seedlings emerged, $\mathrm{CdCl}_{2}$ was added to the culture solution to a final concentration of $50 \mu \mathrm{M}$ for $24 \mathrm{~h}$. Before we 
started cadmium stress, we selected Xi'nong 20 and M1019 plants with the uniform growth for cadmium stress. During sampling, the roots were washed with distilled water and then sampled to remove any remaining $\mathrm{Cd}$ from the surface Roots were individually collected and stored at $-80{ }^{\circ} \mathrm{C}$ for subsequent measurement. Three biological replications were set up in this study and the results and data analysis are based on three biological replications.

\section{Distribution of cd}

Roots were homogenized using a mortar and pestle in a medium containing $250 \mathrm{mM}$ sucrose, $50 \mathrm{mM}$ Tris- $\mathrm{HCl}$ (pH 7.5), and $1 \mathrm{mM}$ dithioerythritol. All steps were performed at $4{ }^{\circ} \mathrm{C}$. The homogenate was centrifuged for 15 min at $3000 \mathrm{rpm}$, and the resulting precipitate included the cell wall components. The supernatant was then centrifuged for $30 \mathrm{~min}$ at $12,000 \mathrm{rpm}$, and the resulting precipitate included the organelle components, and the supernatant comprised the cytoplasmic component [37, 38]. After digesting each component with $\mathrm{HNO}_{3}-\mathrm{HCIO}_{4}$ method, and the $\mathrm{Cd}$ content of each sample was determined by Hitachi Z200.

\section{Cd chemical analysis}

The extraction agents and extraction order were as follows [39]: (1) 80\% ethanol: inorganic salts and amino acids, which are mainly nitrate and chloride acid salt. (2) deionized water: extraction of water-soluble organic acid salts, a generation of heavy metal phosphate. (3) 1 $\mathrm{mol} \cdot \mathrm{L}^{-1}$ sodium chloride solution: extract pectinate, bind with protein or present a heavy metal adsorption state. (4) $2 \%$ acetic acid: the extraction of heavy metal phosphate that is insoluble in water, including secondgeneration phosphate and orthophosphate. (5) 0.6 $\mathrm{mol} \cdot \mathrm{L}^{-1}$ hydrochloric acid: extract oxalate. Extraction method: $2 \mathrm{~g}$ of a fresh sample was placed in a beaker. Approximately $20 \mathrm{~mL}$ of the extraction agent was then added to keep the sample saturated, and recycled extraction after extracting it in a $30{ }^{\circ} \mathrm{C}$ incubator for $18 \mathrm{~h}$. Then, an equal volume of the same extraction agent was added into the beaker of the sample, and recycled extraction for the second time after extract $2 \mathrm{~h}$ and repeat the extraction twice. Finally, each chemical component was extracted 4 times in $24 \mathrm{~h}$, and a total of four extracts $(80 \mathrm{~mL})$ were pooled into a beaker. The extract of the chemical analysis of Cd was evaporated nearly $20 \mathrm{~mL}$ and then was determined using AAS ZEEnit700 after digesting with $\mathrm{HNO}_{3}-\mathrm{HCIO}_{4}$ method.

\section{Protein extraction, trypsin digestion, and tandem mass tags (TMT) labeling}

For digestion, the protein solution was reduced with 5 $\mathrm{mM}$ dithiothreitol for $30 \mathrm{~min}$ at $56^{\circ} \mathrm{C}$ and alkylated with
$11 \mathrm{mM}$ iodoacetamide for $15 \mathrm{~min}$ at room temperature in darkness. The protein sample was then diluted by adding $100 \mathrm{mM}$ TEAB to urea concentration less than 2 M. Finally, trypsin was added at 1:50 trypsin-to-protein mass ratio for the first digestion overnight and 1:100 trypsin-to-protein mass ratio for a second $4 \mathrm{~h}$-digestion.

For digestion, the protein solution was reduced with 5 $\mathrm{mM}$ dithiothreitol for $30 \mathrm{~min}$ at $56^{\circ} \mathrm{C}$ and alkylated with $11 \mathrm{mM}$ iodoacetamide for $15 \mathrm{~min}$ at room temperature in darkness. The protein sample was then diluted by adding $100 \mathrm{mM}$ TEAB to urea concentration less than 2 M. Finally, trypsin was added at 1:50 trypsin-to-protein mass ratio for the first digestion overnight and 1:100 trypsin-to-protein mass ratio for a second 4-h digestion.

After trypsin digestion, the peptide was desalted by Strata X C18 SPE column (Phenomenex) and vacuumdried. The peptide was reconstituted in $0.5 \mathrm{M}$ TEAB and processed according to the protocol provided in the TMT kit. Briefly, one unit of TMT reagent was thawed and reconstituted in acetonitrile [40]. The peptide mixtures were then incubated for $2 \mathrm{~h}$ at room temperature and pooled, desalted, and dried by vacuum centrifugation.

\section{LC-MS/MS analysis and data analysis}

The tryptic peptides were dissolved in $0.1 \%$ formic acid (solvent A), directly loaded onto a home-made reversedphase analytical column (15-cm length, $75 \mu \mathrm{m}$ i.d.). The gradient was comprised of an increase from 6 to $23 \%$ solvent B ( $0.1 \%$ formic acid in $98 \%$ acetonitrile) over 26 min, 23 to $35 \%$ in $8 \mathrm{~min}$ and increasing to $80 \%$ in $3 \mathrm{~min}$, then holding at $80 \%$ for the last $3 \mathrm{~min}$, all at a constant flow rate of $400 \mathrm{~nL} / \mathrm{min}$ on an EASY-nLC 1000 UPLC system. The peptides were subjected to NSI source followed by tandem mass spectrometry (MS/MS) in a $\mathrm{Q}$ Exactive $^{\mathrm{Tm}}$ Plus (Thermo) coupled online to the UPLC. The electrospray voltage applied was $2.0 \mathrm{kV}$. The $\mathrm{m} / \mathrm{z}$ scan range was 350 to 1800 for full scan, and intact peptides were detected in the Orbitrap at a resolution of 70 , 000. Peptides were then selected for MS/MS using NCE setting as 28, and the fragments were detected in the Orbitrap at a resolution of 17,500. A data-dependent procedure that alternated between one MS scan followed by $20 \mathrm{MS} / \mathrm{MS}$ scans with $15.0 \mathrm{~s}$ dynamic exclusion. Automatic gain control (AGC) was set at 5E4. Fixed first mass was set as $100 \mathrm{~m} / \mathrm{z}$.

The secondary mass spectrometry data were retrieved using Maxquant (v1.5.2.8). Parameter Settings: database search for UniProt Triticum estivum (136,892 series), add the library to compute random matching caused by the false-positive rate (FDR), and joined the common pollution in database library, used to eliminate the influence of protein identification results of pollution; The enzyme cutting mode was set to Trypsin/P. The number of missed cut points was set to 2 . The minimum length 
of the peptide segment was set to seven amino acid residues. The maximum number of peptide modification was set to 5 . The quality error tolerance of primary parent ions in First search and Main search was set to 20 ppm; \and $5 \mathrm{ppm}$; $\backslash$, respectively, and that of secondary fragment ions was set to $0.02 \mathrm{Da}$. Cysteine alkylation is set to fixed modification, which can be modified to oxidation of methionine and acetylation of protein $\mathrm{N}$. The quantitative method was set to tmt-6plex, and the FDR for protein identification and PSM identification was set to $1 \%$. The quantitative proteomic analysis was performed using a customer service by PTM-Biolabs Cd., Ltd. (Hangzhou, China).

\section{Abbreviations}

DEPS: Differential expression proteins; MMR: Mismatch repair; TMT: Tandem mass tag; TEAB: Triethyl ammonium bicarbonate; MAPK: Mitogen-activated protein kinase; mRNA: Messenger ribonucleic acid; DNA: Deoxyribonucleic acid; LC-MS / MS: Liquid chromatography tandem mass spectrometry

\section{Acknowledgements}

Not applicable.

\section{Authors' contributions}

MY.J was major writing contributor to the manuscript, DZ. Z was major contributor to analyzing data and text check. Y. Z and YC. H were contributors to acquire and organize data and make a preliminary interpretation to the data. SW. W and XY. W was contributor to drawing and continually modifying Figs. Q. D and LJ. M were contributors to article modification and experimental design. All authors have read and approved the manuscript

\section{Funding}

This study was supported by the "948" Project of Ministry of Agriculture of China (No. 2015Z39, 2016X58), and the National Key Research and Development Project for the 13th Five-year Plan (No. 2016YFD0101602). The funding body was not involved in the design of the study, analysis or interpretation of data or writing the manuscript.

\section{Availability of data and materials}

All data is contained within the manuscript and supplementary material. The datasets generated and analysed during the current study are available in the [ProteomeXchange] repository, [ProteomeXchange accession: PXD018179 and FTP Download: ftp://ftp.pride.ebi.ac.uk/pride/data/archive/2020/03/ PXD018179].

\section{Ethics approval and consent to participate}

Not applicable.

\section{Consent for publication}

Not applicable.

\section{Competing interests}

The authors declare that they have no competing interests.

Received: 4 February 2019 Accepted: 5 April 2020

Published online: 07 May 2020

\section{References}

1. Kosová K, Vítámvás P, Prášil IT, Renaut J. Plant proteome changes under abiotic stress - contribution of proteomics studies to understanding plant stress response. J Proteome. 2011;74(8):1301-22.

2. Kamal AHM, Kihyun K, Kwanghyun S, Jongsoon C, Byungkee B, Tsujimoto H, Hwayoung $H$, Chulsoo P, Sunhee W. Abiotic stress responsive proteins of wheat grain determined using proteomics technique. Aust J Crop Sci. 2010; 4(3):196-208.
3. Marx H, Minogue CE, Jayaraman D, Richards AL, Kwiecien NW, et al. A proteomic atlas of the legume Medicago truncatula and its nitrogen-fixing endosymbiont Sinorhizobium meliloti. Nat Biotechnol. 2016;34(11):1198-205.

4. Li GK, Gao J, Peng H, Shen YO, Ding HP, Zhang ZM, Pan GT, Lin HJ. Proteomic changes in maize as a response to heavy metal (lead) stress revealed by iTRAQ quantitative proteomics. Genet Mol Res. 2016;15(1).

5. Shi Z, Yang S, Han D, Zhou Z, Li X, Liu Y, Zhang B. Silicon alleviates cadmium toxicity in wheat seedlings (Triticum aestivum $L$.) by reducing cadmium ion uptake and enhancing antioxidative capacity. Environ Sci Pollut Res. 2018;25(8):7638-46.

6. Choppala G, Saifullah, Bolan N, Bibi S, lqbal M, Rengel Z, Kunhikrishnan A, Ashwath N, Ok YS. Cellular Mechanisms in Higher Plants Governing Tolerance to cadmium toxicity. Crit Rev Plant Sci. 2014;33(5):374-91.

7. Edgerton MD. Increasing crop productivity to meet global needs for feed, food, and fuel. Plant Physiol. 2009:149(1):7-13.

8. Oh MW, Roy SK, Kamal AHM, Cho K, Cho S, Park C, Choi J, Komatsu S, Woo S. Proteome analysis of roots of wheat seedlings under aluminum stress. Mol Biol Rep. 2014;41(2):671-81.

9. Lu H, Wai CL, Nora FYT, Ye ZH. Effects of root morphology and anatomy on cadmium uptake and translocation in rice (Oryza sativa L.). J Environ Sci. 2019:75(1):299-309.

10. Fahr M, Laplaze L, Bendaou N, Hocher V, Mzibri ME, Bogusz D, Smouni A. Effect of lead on root growth. Front Plant Sci. 2013:4(1):175.

11. Talanova W, Titov AF, Boeva NP. Effect of increasing concentrations of heavy metals on the growth of barley and wheat seedlings. Russ J Plant Physiol. 2001;48(1):100-3.

12. Yang L, Ji J, Harris-Shultz KR, Wang H, Wang H, Abd-Allah EF, Luo Y, Hu X. The Dynamic Changes of the Plasma Membrane Proteins and the Protective Roles of Nitric Oxide in Rice Subjected to Heavy Metal Cadmium Stress. Front Plant Sci. 2016;7:190.

13. Nazar R, lqbal N, Masood A, Khan MIR, Syeed S, Khan NA. Cadmium toxicity in plants and role of mineral nutrients in its alleviation. Am J Plant Sci. 2012 03(10):1476-89.

14. Howden R, Goldsbrough PB, Andersen CR, Cobbett CS. Cadmium-Sensitive, cad7 Mutants of Arabidopsis thaliana are phytochelatin deficient. Plant Physiol. 1995;107(4):1059-66.

15. Balestrasse KB, Noriega GO, Batlle A, Tomaro ML. Heme oxygenase activity and oxidative stress signaling in soybean leaves. Plant Sci. 2006;170(2):33946.

16. Ding $H$, Gao J, Qin $C, M a ~ H$, Huang $H$, Song $P$, Luo $X$, Lin $H$, Shen $Y$, Pan $G$, Zhang Z. The dynamics of DNA methylation in maize roots under $\mathrm{Pb}$ stress. Int J Mol Sci. 2014;15(12):23537-54.

17. Kramer U, Smith RD, Wenzel WW, Raskin I, Salt DE. The role of metal transport and tolerance in nickel Hyperaccumulation by Thlaspi goesingense Hdacsy. Plant Physiol. 1997;115(4):1641-50.

18. Kupper H, Zhao FJ, McGrath SP. Cellular Compartmentation of zinc in leaves of the Hyperaccumulator Thlaspi caerulescens1. Plant Physiol. 1999;119(1): 305-11.

19. Hall JL. Cellular mechanisms for heavy metal detoxification and tolerance. J Exp Bot. 2002;53(366):1-11

20. Hart JJ, Welch RM, Norvell WA, Sullivan LA, Kochian LV, Characterization of cadmium binding, uptake, and translocation in intact seedlings of bread and durum wheat cultivars. Plant Physiol. 1998;118(1):219-26.

21. Kafri M, Metzl-Raz E, Jona G, Barkai N. The cost of protein production. Cell Rep. 2016:14(1):22-31.

22. Ge P, Hao P, Cao M, Guo G, Lv D, Subburaj S, Li X, Yan X, Xiao J, Ma W, Yan $Y$. iTRAQ-based quantitative proteomic analysis reveals new metabolic pathways of wheat seedling growth under hydrogen peroxide stress. PROTEOMICS. 2013;13(20):3046-58.

23. Banerjee S. Cadmium inhibits mismatch repair by blocking the ATPase activity of the MSH2-MSH6 complex. Nucleic Acids Res. 2005;33(4):1410-9.

24. Aina R, Labra M, Fumagalli P, Vannini C, Marsoni M, Cucchi U, Bracale M, Sgorbati S, Citterio S. Thiol-peptide level and proteomic changes in response to cadmium toxicity in Oryza sativa L. roots. Environ Exp Bot. 2007; 59(3):381-92.

25. Liu W, Yang YS, Francis D, Rogers HJ, Li P, Zhang Q. Cadmium stress alters gene expression of DNA mismatch repair related genes in Arabidopsis seedlings. Chemosphere. 2008:73(7):1138-44.

26. Jin YH, Clark AB, Slebos RJC, Al-Refai H, Taylor JA, Kunkel TA, Resnick MA, Gordenin DA. Cadmium is a mutagen that acts by inhibiting mismatch repair. Nat Genet. 2003;34(3):326-9. 
27. Daugaard $M$, Rohde $M$, Jäättelä $M$. The heat shock protein 70 family: highly homologous proteins with overlapping and distinct functions. FEBS Lett. 2007;581(19):3702-10.

28. Lewis S, Donkin ME, Depledge MH. Hsp70 expression in Enteromorpha intestinalis (Chlorophyta) exposed to environmental stressors. Aquat Toxicol. 2001;51(3):277-91.

29. Wiseman RL, Powers ET, Buxbaum JN, Kelly JW, Balch WE. An adaptable standard for protein export from the endoplasmic reticulum. Cell. 2007; 131(4):0-821.

30. Batey RT, Doudna JA. Structural and energetic analysis of metal ions essential to SRP signal recognition domain assemblyt. Biochemistry. 2002; 41(5):11703-11710.31.

31. Park SKJF. Functional analysis of the signal recognition particle in Escherichia coli by characterization of a temperature-sensitive ffh mutant. J Bacteriol. 2002;184(10):2642-53.

32. Hasona A, Crowley PJ, Levesque CM, Mair RW, Cvitkovitch DG, Bleiweis AS, Brady LJ. Streptococcal viability and diminished stress tolerance in mutants lacking the signal recognition particle pathway or YidC2. Proc Natl Acad Sci U S A. 2005;102(48):17466-71

33. Althoff SM, Stevens SW, Wise JA. The Srp54 GTPase is essential for protein export in the fission yeast Schizosaccharomyces pombe. Mol Cell Biol. 1994; 14(12):7839-54.

34. Kalies KU, Rapoport TA, Hartmann E. The beta subunit of the Sec61 complex facilitates cotranslational protein transport and interacts with the signal peptidase during translocation. J Cell Biol. 1998;141(4):887-94.

35. Edwards R, Dixon DP, Walbot V. Plant glutathione S-transferases: enzymes with multiple functions in sickness and in health. Trends Plant Sci. 2000;5(5): 193-8.

36. Do $T H T$, Martinoia $E$, Lee $Y$. Functions of $A B C$ transporters in plant growth and development. Curr Opin Plant Biol. 2018;41:32-8.

37. Weigel HJ, Jäger HJ. Subcellular distribution and chemical form of cadmium in bean plants. Plant Physiol. 1980;65(3):480-2.

38. Wang $X$, Liu $Y$, Zeng G, et al. Subcellular distribution and chemical forms of cadmium in Bechmeria nivea (L.) gaud. Environ Exp Bot. 2008;62(3):389-95.

39. Wu FB, Dong J, Qian QQ, et al. Subcellular distribution and chemical form of $\mathrm{cd}$ and $\mathrm{cd}-\mathrm{Zn}$ interaction in different barley genotypes. Chemosphere. 2005;60(10):0-1446.

40. Plubell DL, Wilmarth PA, Zhao Y, et al. Extended multiplexing of tandem mass tags (TMT) labeling reveals age and high fat diet specific proteome changes in mouse Epididymal adipose tissue. Mol Cell Proteomics. 2017; 16(5):873-90.

\section{Publisher's Note}

Springer Nature remains neutral with regard to jurisdictional claims in published maps and institutional affiliations.

Ready to submit your research? Choose BMC and benefit from:

- fast, convenient online submission

- thorough peer review by experienced researchers in your field

- rapid publication on acceptance

- support for research data, including large and complex data types

- gold Open Access which fosters wider collaboration and increased citations

- maximum visibility for your research: over $100 \mathrm{M}$ website views per year

At $\mathrm{BMC}$, research is always in progress.

Learn more biomedcentral.com/submissions 